\title{
Composite Scrambling Technique to Combat PAPR by Combining SLM with Signal Distortion Methods
}

\author{
N.Hariprasad \\ Research Scholar, \\ Sathyabama University, \\ Chennai, India,
}

\author{
Alamelu Nachiappan,Ph.D \\ Associate Professor, \\ Pondicherry Engineering College, \\ Pondicherry, India,
}

\begin{abstract}
Peak to average Power Ratio (PAPR) is the major drawback of orthogonal Frequency Division Multiplexing (OFDM) systems. Since OFDM is the indispensable technology for $4 \mathrm{G}$ wireless schemes, great attention is given to PAPR reduction. Some of the techniques classified under signal distortion and signal scrambling already existing to meet the needs, but their effectiveness is still a question for research. In this background this paper presents a new -composite scrambling-technique which combines SLM, clipping and peak windowing methods. Comparative analysis of this proposed system with different PAPR reduction techniques are done and the advantages are discussed with their tread offs. MATLAB programming is used for simulation.
\end{abstract}

\section{Keywords:}

OFDM, PAPR, SLM. Peak Windowing, Clipping, complementary cumulative distribution function (CCDF).

\section{INTRODUCTION}

In present day broadband wireless systems of $4 \mathrm{G}$ the key performer is orthogonal frequency division multiplexing (OFDM). Considering its effectiveness many of the IEEE standard working groups (example: IEEE802.16d/e, 802.15.3a, $802.11 \mathrm{a} / \mathrm{g} / \mathrm{n}$ ) also approved OFDM. The major practical limiting factor of the performance of OFDM is the Peak to Average Power Ratio (PAPR). The large signal peaks of the time domain signal demands for a power amplifier that has wide linear dynamic region. Otherwise the resulting nonlinear distortion drives the amplifier to saturation region, which produces corrupted signal due to interference among the subcarriers. To reduce PAPR if we alter the average power then the system's Bit Error Rate (BER) performance will be degraded. This is undesirable. So the remaining option is to reduce the peak power. Many techniques for reducing peak power and hence the PAPR have been proposed[1,2,3]. They can be broadly categorised in to three groups as 1) signal distortion methods 2)Signal scrambling methods, and 3)Coding methods.

In this paper, as a combination of some of the methods of above said categories, a new _composite scrambling technique is proposed. Comparative

analysis of the proposed technique with the existing methods is carried overand the details are furnished in the following sections. For this, the paper is organised as follows. Section 2 briefs the technical details of thePAPR in OFDM. Section 3 discusses about the different categories of PAPR reduction techniques. Proposed composite scrambling technique is explained in section 4 .Section 5 deals with simulation results and section 6 is conclusion and future work.

\section{PAPR OF THE OFDM}

If $x(t)$ is the continuous time OFDM signal the PAPR of $x(t)$ is defined as the ratio of the maximum (peak) instantaneous power to that of the average power.

$$
\operatorname{PAPR}[x(t)]=\frac{\operatorname{Max}_{0 \leq \mathrm{t} \leq \mathrm{NT}}[\mathrm{x}(\mathrm{t})]^{2}}{\mathrm{P}_{\text {avg }}} \text { (1) }
$$

$\mathrm{P}_{\mathrm{avg}}$ is the average power of the OFDM signal $\mathrm{x}(\mathrm{t})$.that can be calculated in frequency domain. For discrete-time OFDM also PAPR is calculated, which determines the complexity of the digital circuitry in terms of number of bits necessary to achieve desired signal to noise ratio.[3] But PAPR of the continuous signal practically finds more importance as its reduction reduces the power dissipation and cost of the components used.

\section{PAPR REDUCTION TECHNIQUES}

Clipping is the simplest technique used to reduce PAPR. As per this signal distortion technique a limiting threshold level (L) will be fixed by selecting a clipping ratio. Any signal with amplitude level below this $\mathrm{L}$ will be allowed as it is and the signal level that exceeds the $\mathrm{L}$ will be trimmed-off to $\mathrm{L}$. Clipping mathematically expressed as follows,

$$
\begin{aligned}
& \mathrm{x},|\mathrm{x}| \leq \mathrm{L} \\
& \mathrm{A}(\mathrm{x})=\mathrm{L},|\mathrm{e}|>\mathrm{L} \quad \mathrm{L} \text { is a positive real number }
\end{aligned}
$$

In this the clipping is performed upon the amplitude of the time domain signal while its phase remains unaltered. In band distortion and Out of band radiation are the two undesirable side effects of clipping, the first-one degrades the bit error rate performance and the next leads to reduction in spectral efficiency. In band distortion is the clipping noise introduced in the spectrum which can't be removed with any filtering. This aliasing problem can be avoidedonly byover sampling. That means implementing zero padding with each OFDM block and to take long IFFT.[4]. Out of band radiation is the expansion of spectrum outside the limit. This out of band noise can be removed by filtering, but again the trade-off is peak regrowth. To avoid peak regrowth repeated clipping and filtering should be done [5],which increases the system complexity, .The block diagram of the clipping procedure after zero padding is as shown in the fig 1 . 


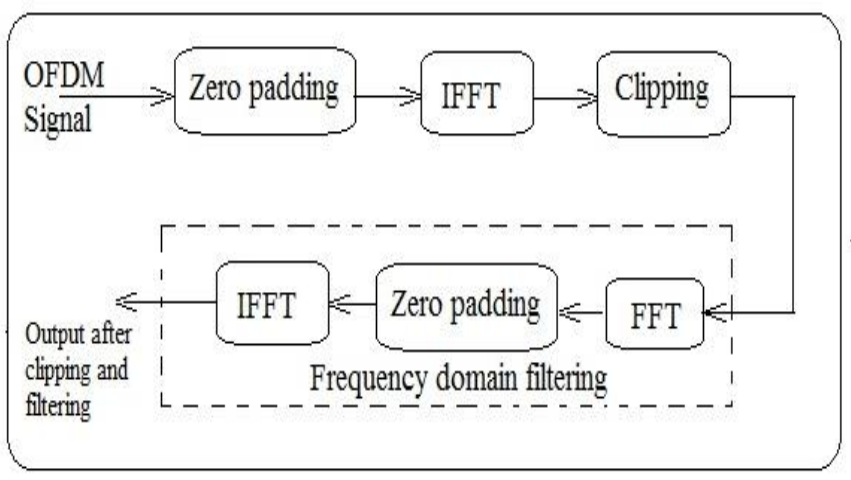

Fig. 1 clipping after zero padding

Peak Windowing is the other signal distortion technique which can render better performance than clipping [6][8]. According to this procedure a window function, usually with better spectral properties is multiplied with the OFDM envelop. The desired spectral characteristics are,

1. Narrow band - As much as the band is slim the out of band interference is mitigated.

2. Optimum length in time demine - Ensures less interference with other samples (otherwise Bit error rate will be greatly affected.)

Considering these points Hamming window, Kaiser window, Blackman window are some of the best suited functions for peak windowing [4]. The block diagram of the peak windowing process is shown in fig 2 .

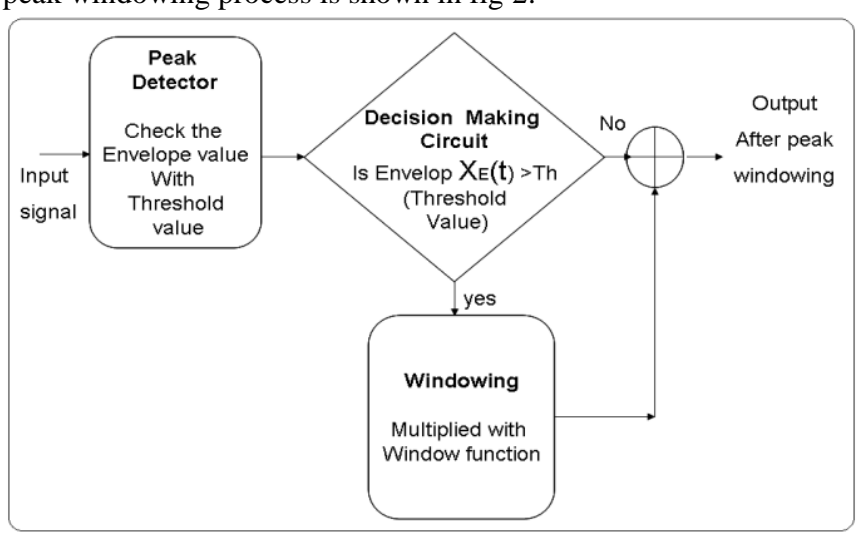

Fig. 2 Peak windowing process

Peak detector is the circuit which analyses the OFDM envelop and compares its peak value $X_{\text {En }}(t)$ with a threshold value (Th).If the value of the envelop found to be exceeding the $\mathrm{Th}$, the decision making circuit directs it to windowing block. Here it gets multiplied with the windowing function.So, $\mathrm{X}_{\mathrm{En}}(\mathrm{t})$ becomes $\tilde{\mathrm{X}}_{\mathrm{En}}(\mathrm{t})$.

Where $\tilde{X}_{\text {En }}(\mathrm{t})=\mathrm{X}_{\mathrm{En}}(\mathrm{t}) \cdot\left[1-\sum \phi \cdot \omega_{n}(t-\hat{t})\right]$

$\hat{t}$ is the position of the threshold of the envelop

$\phi$ is the attenuation constant

$\omega_{n}(t)$ is the window function

For rest of the portions where peak value less than that of the threshold, windowing is not applied. The resulting signal at the output of the peak windowing process is almost free from the peaks. The out of the OFDM signal after clipping and filtering can be given as input to this peak windowing. In such case the out of band distortion of the clipped signal can be verymuch reduced.

Selective Mapping (SLM) Technique is another major technique popularly used to reduce PAPR. This is a signal scrambling technique in whichmultiple OFDM frames representing the same information will be generated and out of them,one with minimum PAPR is selected and transmitted.

Let there be $\mathrm{N}$ orthogonal subcarriers in an OFDM system, which are used to transmit modulation symbols $\mathrm{x}_{\mathrm{n}}$ of complex nature. $\mathrm{N}$ such symbols each occupying a subcarrier constitutes a data vector $\mathrm{X}=\left(\mathrm{x}_{\mathrm{n}}\right)$ N.The data block is given by $\mathrm{X}=\left[\mathrm{X}_{0}, \mathrm{X}_{1}, \mathrm{X}_{2}, \ldots \mathrm{X}_{\mathrm{N}-1}\right]^{\mathrm{T}}$

Let $B^{(v)}=\left[B_{0}^{(v)}, B_{1}^{(v)}, B_{2}^{(v)}, \ldots . B_{N-1}{ }^{(v)}\right]^{T} \quad V$ phase sequences of statistically independent nature are generated in which $\mathrm{v}=1,2,3 \ldots \mathrm{V}, \mathrm{B}_{\mathrm{k}}{ }^{(\mathrm{v})}=\exp \left(\mathrm{j} \Phi_{\mathrm{k}}{ }^{(\mathrm{v})}\right), \mathrm{k}=1,2,3 \ldots \mathrm{N}-1 \quad$, and $\Phi_{\mathrm{k}}{ }^{(\mathrm{v})} \in$ $[0,2 \pi]$. Then the data block $X$ is multiplied with the $\mathrm{V}$ vectors in an 'element by element' manner. This gives rise to $X^{(v)}$ where,

$\mathrm{X}^{(\mathrm{v})}=\left[\mathrm{X}_{0} \mathrm{~B}_{0}{ }^{(\mathrm{v})}, \mathrm{X}_{1} \mathrm{~B}_{1}{ }^{(\mathrm{v})}, \mathrm{X}_{2} \mathrm{~B}_{2}{ }^{(\mathrm{v})}, \ldots \mathrm{X}_{\mathrm{N}-1} \mathrm{~B}_{\mathrm{N}-1}{ }^{(\mathrm{v})}\right]^{\mathrm{T}}$, Where $\mathrm{v}=1,2,3 \ldots \mathrm{V}$. This process is clearly depicted in the fig 3 .

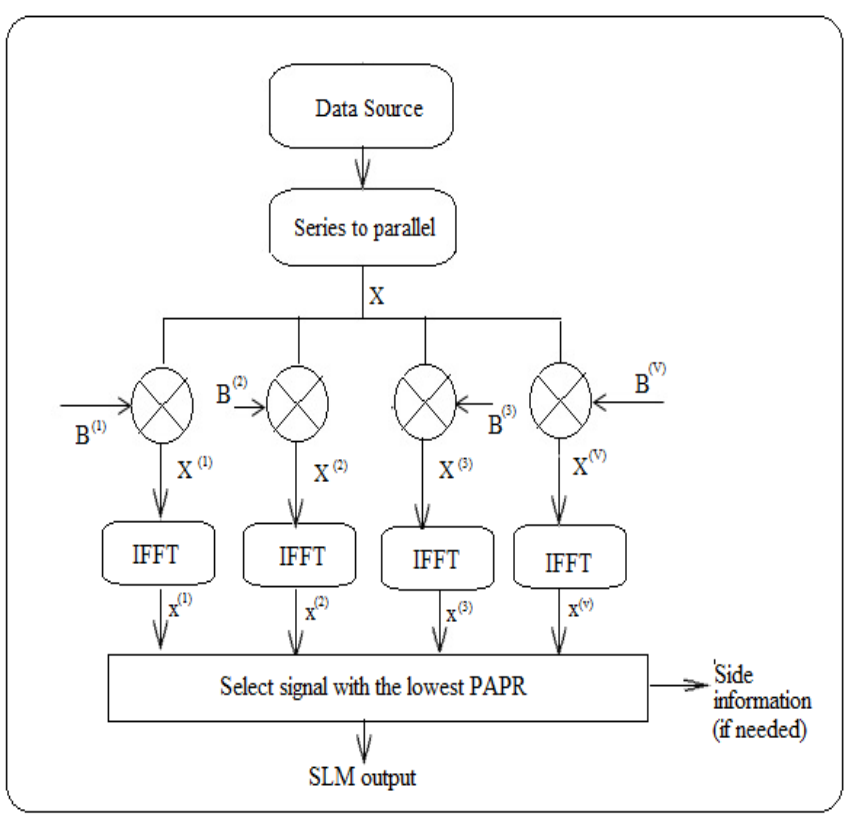

Fig. 3 Block diagram of selective mapping

Later IFFT is performed which transforms the $\mathrm{X}^{(\mathrm{v})}$ into time domain, to get $\mathrm{x}^{(\mathrm{v})}$, where $\mathrm{v}=1,2,3 \ldots \mathrm{V}$. Finally from this $x^{(v)}$,the signal with minimum PAPR is selected for transmission. Out of $\mathrm{V}$ signals, which signal was selected for transmission is needed to be informed to the receiver side. For this $\log _{2} \mathrm{~V}$ bits are additionally required in case of binary symbol transmission. This is said to be the side information. With some additional complexity or coding the side information may be dropped [7].

\section{COMPOSITE SCRAMBLING TECHNIQUE}

In this proposed method Selective mapping technique is applied and the resulting signal with the lowest PAPR under goes a series of signal distortion techniques. First clipping is 
performed with a selectable clipping ratio $C_{R}$. The ratio of the threshold voltage for clipping to that of the square root of the average power of the OFDM is said to be the clipping ratio. With this selectable $C_{R}$. we have more control over the selection of lowest PAPR. Next comes the peak windowing block. The window function used in this technique is Black man window, which is given by

$$
w(n)=a_{0}-a_{1} \cos \left(\frac{2 \pi n}{N-1}\right)+a_{2} \cos \left(\frac{4 \pi n}{N-1}\right)
$$

Where

$a_{0}=0.42659, a_{1}=0.49656, a_{2}=0.076849$ and $\mathrm{N}$ is the number of subcarriers. Comparing Hamming window, here Black man window is selected because the additional cosine term comparatively reduces more out of band radiation. So the adverse effect of clipping is removed and the PAPR is further reduced. In the total process so far, reduction of peak power is considered. To reduce the PAPR, increase in average power also plays some role. That part is taken care by the next stage (refer fig 4) the pre amplification block.

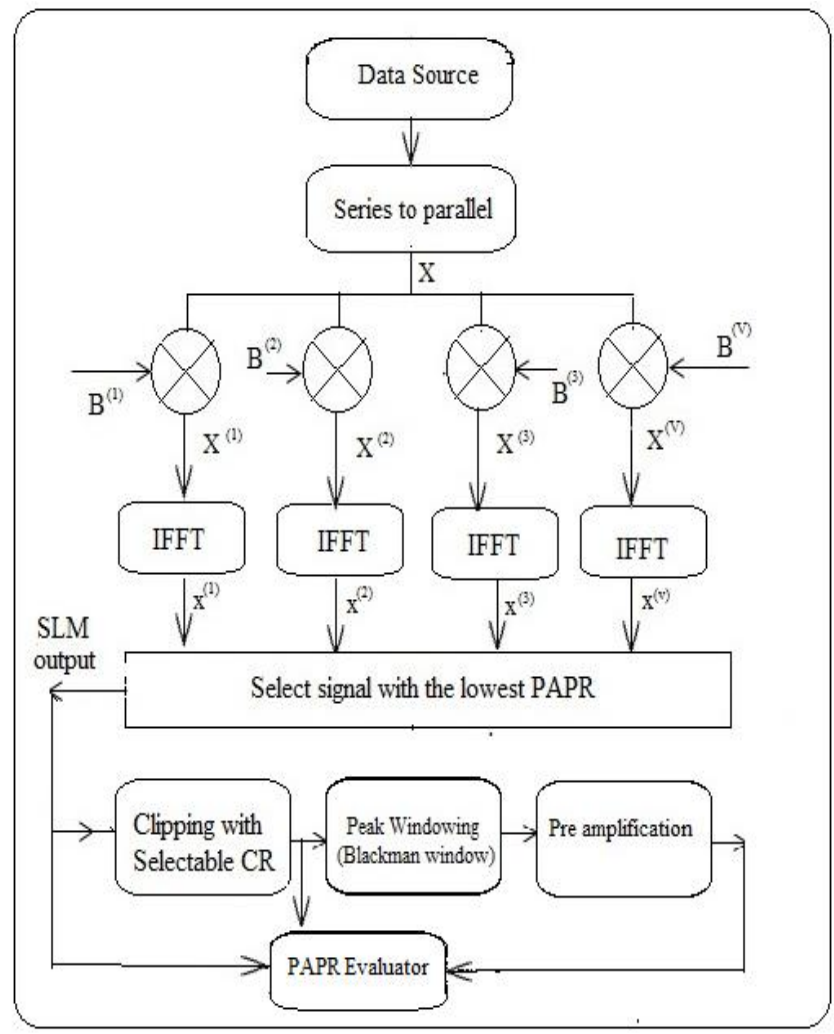

Fig. 4 Block diagram of Composite scrambling technique

In the windowing process signal above the threshold level is considered. The signal below the threshold is taken for pre amplification. This improves the average power and thereby the PAPR is further reduced.

\section{SIMULATION RESULTS}

The proposed composite scrambling technique is simulated using MATLAB program. Theparameters taken for simulation arelisted in table1 figure wise.

\begin{tabular}{|l|l|l|}
\hline \multicolumn{3}{|c|}{ Table 1.simulation parameters } \\
\hline Parameters & $\begin{array}{l}\text { clipping ratio } \\
(\mathrm{CR})\end{array}$ & $\begin{array}{l}\text { constellation } \\
\text { size(CS) }\end{array}$ \\
\hline Fig 5 & Above 55\% & 8 \\
\hline Fig 6 & Above 55\% & 16 \\
\hline Fig 7 & Above 60\% & 8 \\
\hline Fig 8 & Above 60\% & 16 \\
\hline Fig 9 & Above 65\% & 8 \\
\hline Fig 10 & Above 65\% & 16 \\
\hline
\end{tabular}

For all the simulations the unaltered parameters areshown in table 2 ,

\begin{tabular}{|l|l|}
\hline \multicolumn{2}{|c|}{ Table 2.Unaltered parameters } \\
\hline Parameter & Type/Value \\
\hline Modulation & M-PSK \\
\hline Over Sampling & 4 \\
\hline No of data points & 64 \\
\hline No of FFT/IFFT size & 128 \\
\hline No of OFDM symbols & 1000 \\
\hline No of phase matrix generated & 6 \\
\hline
\end{tabular}

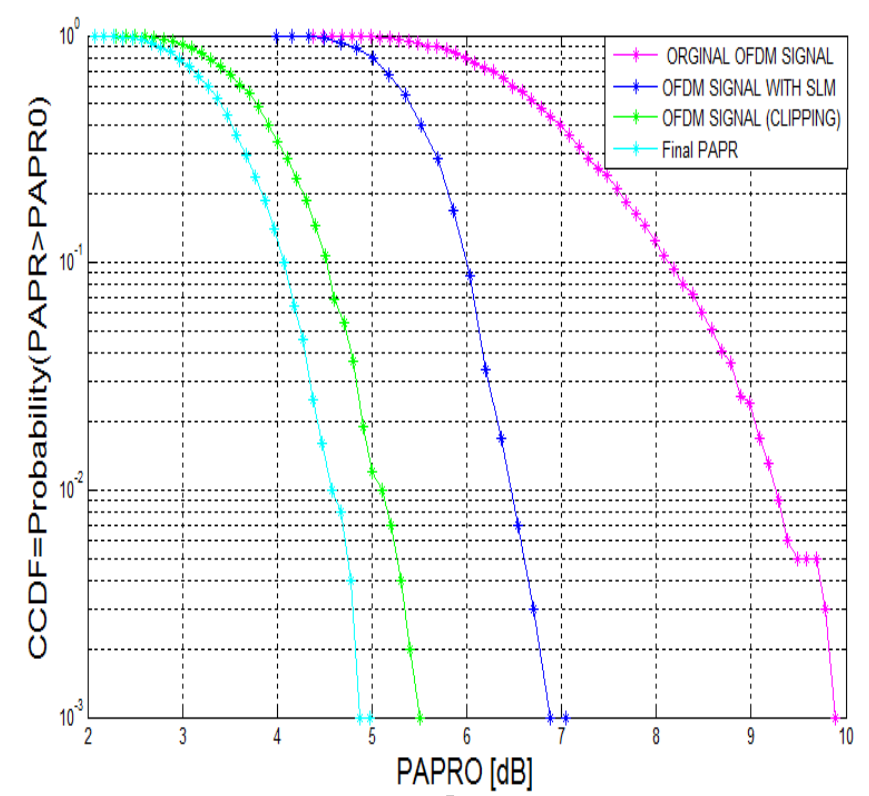

Fig. 5 PAPRO Vs CCDF for $\mathrm{CR}=55 \%, \mathrm{CS}=8$ 
Volume 72- No.10, June2013

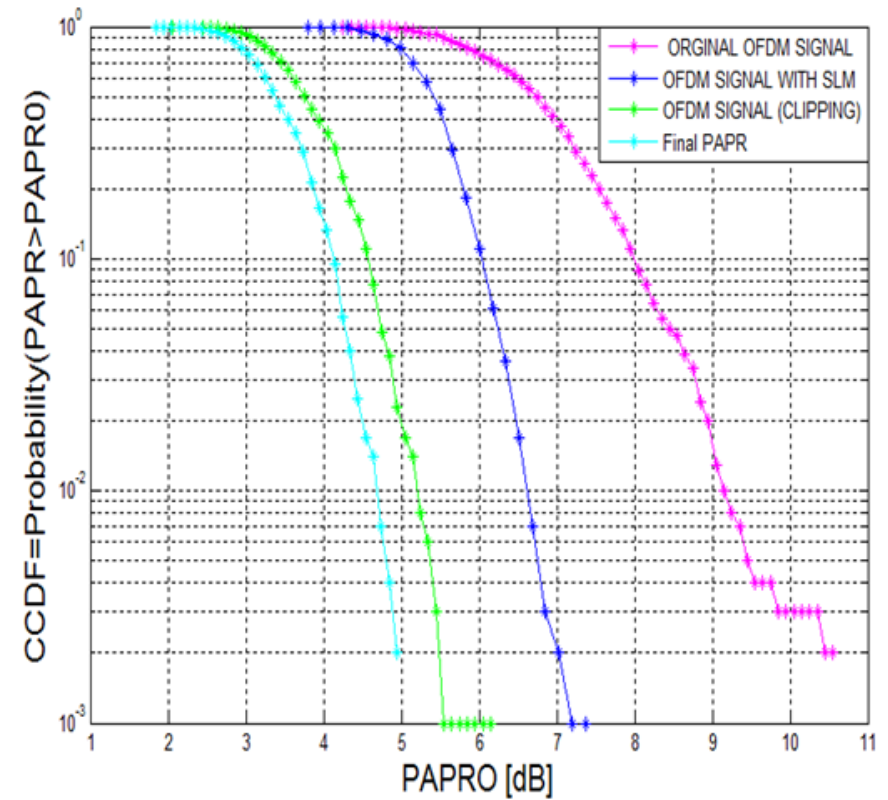

Fig. 6 PAPRO Vs CCDF for $\mathrm{CR}=55 \%, \mathrm{CS}=16$

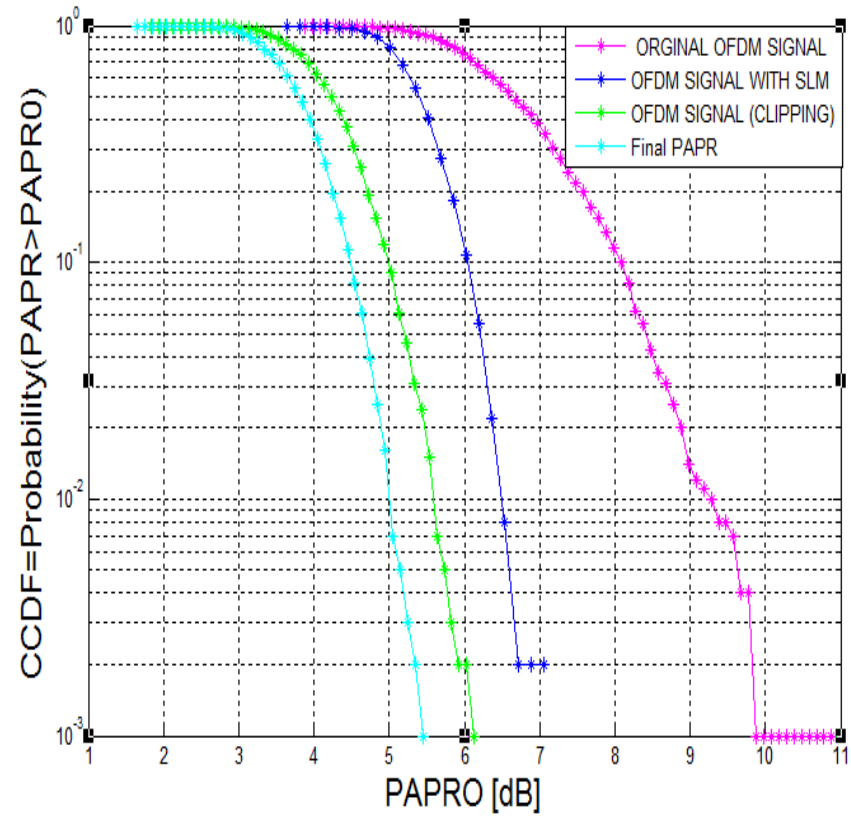

Fig. 7 PAPRO Vs CCDF for $\mathrm{CR}=60 \%, \mathrm{CS}=8$

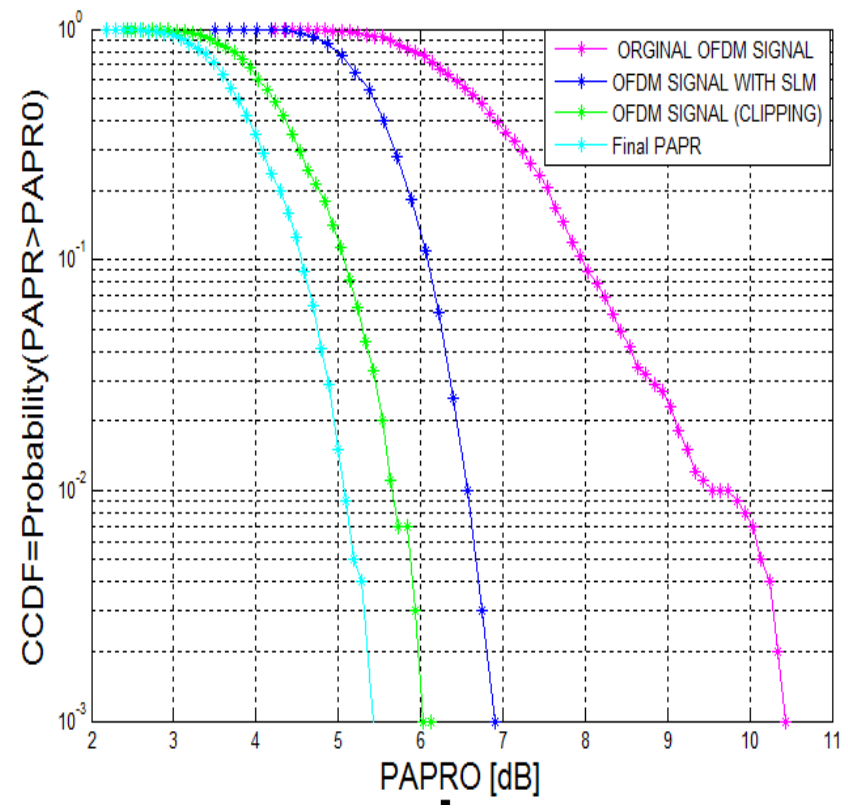

Fig. 8 PAPRO Vs CCDF for $\mathrm{CR}=60 \%, \mathrm{CS}=16$

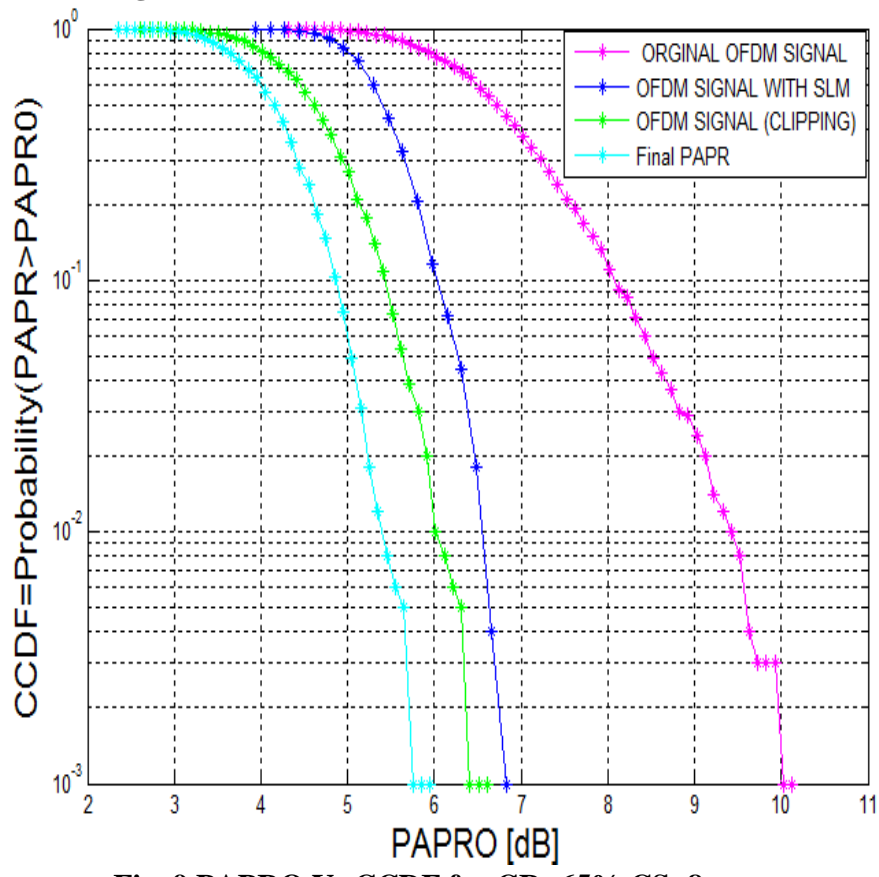

Fig. 9 PAPRO Vs CCDF for $\mathrm{CR}=65 \%, \mathrm{CS}=8$

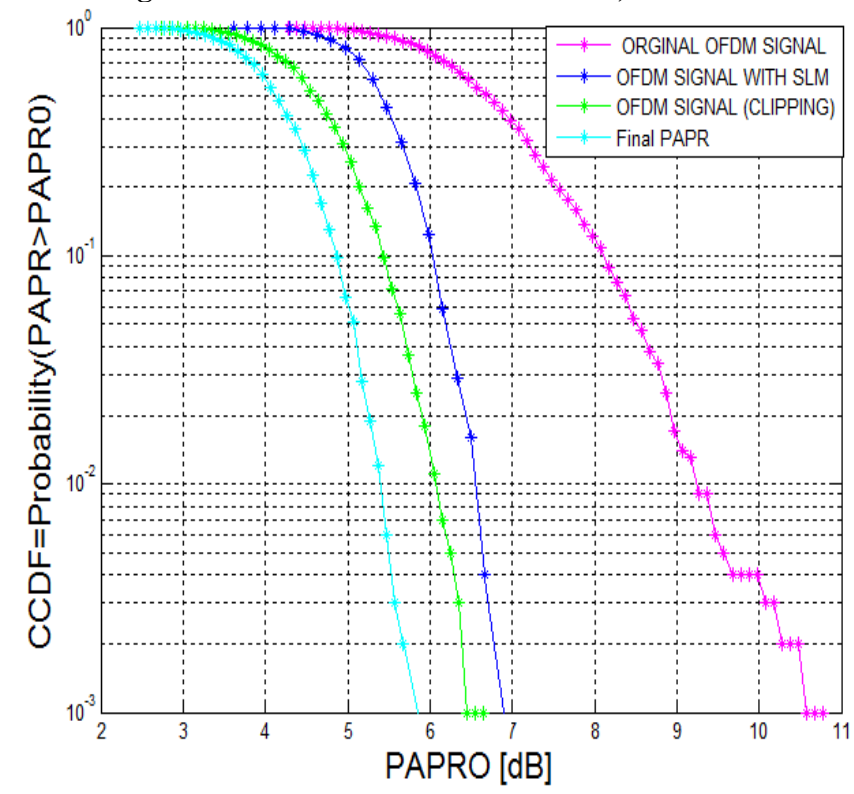

Fig. 10 PAPRO Vs CCDF for $\mathrm{CR}=65 \%, \mathrm{CS}=16$

Figures 5 through 10 shows the CCDF curves of OFDM under various PAPR reduction schemes. The curves for 'original OFDM' refers to the CCDF of randomly generated OFDM samples with no PAPR reduction technique applied. 'OFDM with SLM' is the CCDF after applying selective mapping technique over the OFDM signal. In this case by varying the size of the phase matrix the reduction level can be varied to a great extant. But our prime aim in this paper is to show the effect of the composite scrambling scheme, so the phase matrixvalue is fixed as 6. The next CCDF curve is obtained after clipping. The clipping ratio is specified as the percentage of the maximum value. For three different levels of clipping,two constellations of 8 and 16 yields six CCDF plots. In these plots the 'final PAPR' is the reduced PAPR after applying peak windowing and pre amplification. 


\section{CONCLUSIONS}

The proposed composite scrambling technique presented in this paper is a combination of SLM, clipping, and peak windowing methods. In all the methodsif individuallycarried over that will be aiming on the peak reduction only and thereby ending up with side effects like in band distortion, out of band radiation etc. Here the PAPR of SLM output is reduced by clipping and the out of band radiation because of clipping is combated by Peak windowing. After that the signal below the windowing threshold is pre amplified so that the average power of the signal gets increased and hence PAPR obtained at the lowest value. Considering any single PAPR reduction scheme applied alone, this new scheme outperforms it fairly by a good margin. Slight computational complexity is the price paid as the tradeoff.

For future work the single clipping block can be replaced with repeated clipping and filtering blocks. The phase matrix value fixed in our case can be replaced with a phase rotation scheme. By changing the window function also the desired improved results may be obtained. In that process overcoming / tackling the additional computational complexity needs further research.

\section{REFERENCES}

[1] Bauml, R.W., Fischer, R.F.H., and Huber, J.B. (1996) Reducing the peak-to-average power ratio of multicarrier modulation by selective mapping. Electron.Lett., 32(22), pp.2056-2057

[2] Cimini, L.J. Jr (2000) Peak-to-average power ratio reduction of anOFDMsignal using partial transmit sequences.IEEE Commun. Letters, 4(3), pp.86-88.
[3] Tao Jiang and Yiyan Wu,(2008) An Overview: Peakto-Average Power Ratio Reduction Techniques for OFDM Signals,IEEE TRANSACTIONS ON BROADCASTING, 54 (2), pp.257-268

[4] Madhusmita Mishra, sarat Kumar Patra and ashokkumarTuruk(2011), "Analysis of Peak to average powerreduction techniques for OFDM using a New Phase sequence." International journal for computer Applications, 35 (6), pp.52-56

[5] J. Armstrong,(2002) "Peak-to-average power reduction for OFDM by repeated clipping and frequency domain filtering" Electron. Lett.,38(5),pp.246-247

[6] V. Vijayarangan, DR. (MRS) R. Sukanesh, "AnOverview of techniques forreducing peak to average power ratio and its selection criteria for orthogonal frequency division multiplexing radio systems.", Journal of Theoretical and Applied Information Technology,(2005-2009) ,pp.25-36

[7] Marco Breiling, Stefan H. Müller-Weinfurtner, and Johannes B. Huber, "SLM Peak-Power

Reduction Without Explicit Side Information" IEEE COMMUNICATIONS LETTERS,(2001) 5, (6),pp.239-241

[8] Guoguang Chen, Rashid Ansari, Yingwei Yao (2009), "Improved Peak Windowing for PAPRReduction in OFDM".IEEE 